\title{
Myoclonies du voile associées à une diminution de l'acide 5-hydroxy-indole-acétique céphalo-rachidien et répondant au clonazépam
}

\author{
S. GAUTHIER, S.N. YOUNG et D.W. BAXTER
}

SUMMARY: $A$ patient presenting with palatal myoclonus was diagnosed as suffering from possible multiple sclerosis. A reduction of cerebrospinal 5-hydroxyindole-acetic acid suggested a decreased central serotonergic turnover. $A$ therapeutic trial with clonazepam, a serotonergic agonist, improved the myoclonus symptomatically. The possible involvement of serotonergic inhibitory systems in the expression of palatal myoclonus is discussed.

RÉSUME: Une patiente présentant des myoclonies du voile a été diagnostiquée comme souffrant de sclérose en plaques possible. Une réduction de l'acide 5hydroxy-indole-acétique céphalo-rachidien a suggéré une diminution du turnover sérétonergique central. Un essai thérapeutique au clonazépam, un agoniste sérotonergique central. Un essai thérasymptomatique des myoclonies. La possibilité que certains systèmes sérotonergiques inhibiteurs soient impliqués dans l'expression des myoclonies du voile est discutée.

De l'Institut et Hôpital Neurologique de Montréal, Département de Neurologie et de Neurochirurgie, Université McGill et le Département de Psychiatrie, Université McGill.

Tirés à part: Dr. Serge Gauthier, Institut Neurologique de Montréal, 3801, rue Université, Montréal, Québec, Canada H3A 2B4.

Reprint requests to Dr. Serge Gauthier, Institut Neurologique de Montréal, 3801, rue Université, Montréal, Québec, Canada H3A 2B4.
Le syndrome des myoclonies du voile constitue l'expression d'une lésion de voies anatomiques bien définies. Nous rapportons dans cette communication des données nouvelles concernant un neurotransmetteur potentiellement impliqué dans ce syndrome.

\section{OBSERVATION}

Il s'agit d'une femme de 45 ans qui présente depuis 5 ans une dysarthrie progressive. Diagnostiquée comme souffrant de "dysphonie spastique" il y a deux ans, elle subit une résection du nerf récurrent laryngé gauche. Il s'ensuivit une légère amélioration de la voix, qui n'a pas changé depuis.

Plus tard la même année, la patiente s'est plainte de dysphagie aux aliments solides, et une première investigation neurologique révéla des myoclonies régulières du palais mou affectant également le pharynx, le larynx et la corde vocale droite, à un rythme de 130 par minute. Les analyses de routine, incluant une tomodensitométrie cérébrale et une ponction lombaire, furent normales. Aucun traitement spécifique ne fut donné.

Par la suite, la patiente a rapporté une fatigabilité de la jambe gauche, un tremblement intermittent du bras gauche, une diminution de la sensation tactile à l'hémicorps gauche, et une diplopie au regard latéral gauche. Elle fut admise à l'Institut Neurologique de Montréal pour réévaluation.

Les antécédents médicaux pertinents ont révélé une perte de vision monoculaire gauche pendant quelques heures au début de la vingtaine, puis de la diplopie intermittente pendant la première grossesse. Il y a présence d'une surdité non progressive du côté gauche, datant de l'enfance, et il y a eu une rougeole non compliquée à l'âge de 22 ans. Les antécédents familiaux sont sans particularités.

L'examen neurologique a démontré des signes moteurs discrets à l'hémicorps gauche avec diminution du pli nasolabial, lenteur des mouvements alternatifs rapides de la main et du pied, et de la difficulté à sauter sur la jambe. Un léger tremblement d'attitude aux deux bras fut noté. Les réflexes ostéotendineux étaient symétriques, mais la réponse plantaire équivoque à gauche. L'examen sensitif a démontré une diminution du tact léger à l'hémicorps gauche, incluant l'hémiface. La surdité à gauche et les myoclonies rythmiques du voile s'étendant à toutes les structures du cou furent notées telles que décrites plus haut.

Un examen neuro-ophtalmologique détaillé a démontré que l'acuité visuelle, la perception des couleurs, les champs visuels au périmètre de Goldman, les fonds d'oeil et les réponses des pupilles étaient entièrement normaux. Les mouvements de poursuite oculaire étaient saccadiques avec un nystagmus horizontal du type parésie du regard conjugué (gaze paretic), ainsi qu'un nystagmus de rebond au retour à la ligne médiane. L'électrorétinogramme ainsi que les potentiels évoqués visuels étaient normaux.

Un examen otorhinolaryngologique confirma la présence de myoclonies de la corde vocale droite (la gauche étant paralysée chirurgicalement) synchrones avec le palais mou.

Les potentiels évoqués somatosensitifs par stimulation du nerf sural gauche avaient une latence prolongée et une morphologie anormale, en comparaison avec les réponses évoquées par stimulation à droite.

Les radiographies simples du crâne et des poumons étaient normales. Une encéphalographie gazeuse a démontré une atrophie midvermienne, sans 
anomalie notable du tronc cérébral (figure 1).

Tel que décrit récemment (Young et al., 1980), des échantillons de liquide céphalo-rachidien (LCR) furent prélevés au tout début et à la fin de linjection des $100 \mathrm{cc}$ d'oxygène requis pour l'encéphalographie gazeuse. Ces deux échantillons correspondent à deux compartiments bien distincts du LRC, lombaire et cisternal respectivement. Les niveaux d'acide 5-hydroxyindole-acétique (5HIAA) et de tryptophane furent mesurés dans les deux échantillons par la méthode d'Anderson et Purdy (1979). Une diminution notable du SHIAA est notée dans le compartiment lombaire, ainsi qu'une diminution du tryptophane, par rapport à des contrôles du même sexe, d'âge équivalent, ainsi qu'à trois cas de sclérose en plaques possible, soumis à l'encéphalographie gazeuse pour fin diagnostique (tableau 1). Le LRC fut également analysé pour la protéinorachie (13 $\mathrm{mg} \%$ ), la fraction d'immunoglobulines $\mathrm{G}(4 \%)$, et la protéine basique de la myéline $(11 \mathrm{ng} / \mathrm{ml}$ légèrement positive). La cytologie du LCR démontra une légère augmentation non spécifique du sédiment en cellules mononucléaires.

L'impression clinique étant celle de "sclérose en plaques possible", un traitement symptomatique des myoclonies du voile avec le clonazépam, $0.5 \mathrm{mg}$ deux fois par jour, fut commencé. Un mois plus tard, la voix de la patiente est plus ferme et audible; à l'examen clinique, les myoclonies ont nettement diminué en intensité, et la fréquence est plus lente et irrégulière.

\section{COMMENTAIRE}

Etant donné l'histoire antérieure de troubles neurologiques transitoires et la présence d'au moins une lésion localisée au tronc cérébral, sans évidence de lésion vasculaire ou expansive locale, le diagnostic de "sclérose en plaques possible" a été formulé selon les critères de McAlpine et al (1972).

Parmi les facteurs étiologiques des myoclonies du voile, la sclérose en plaques est rare, Rondot et Ben Hamita n'ayant retrouvé que cinq observations cliniques dans la littérature (1968). Cependant, les voies

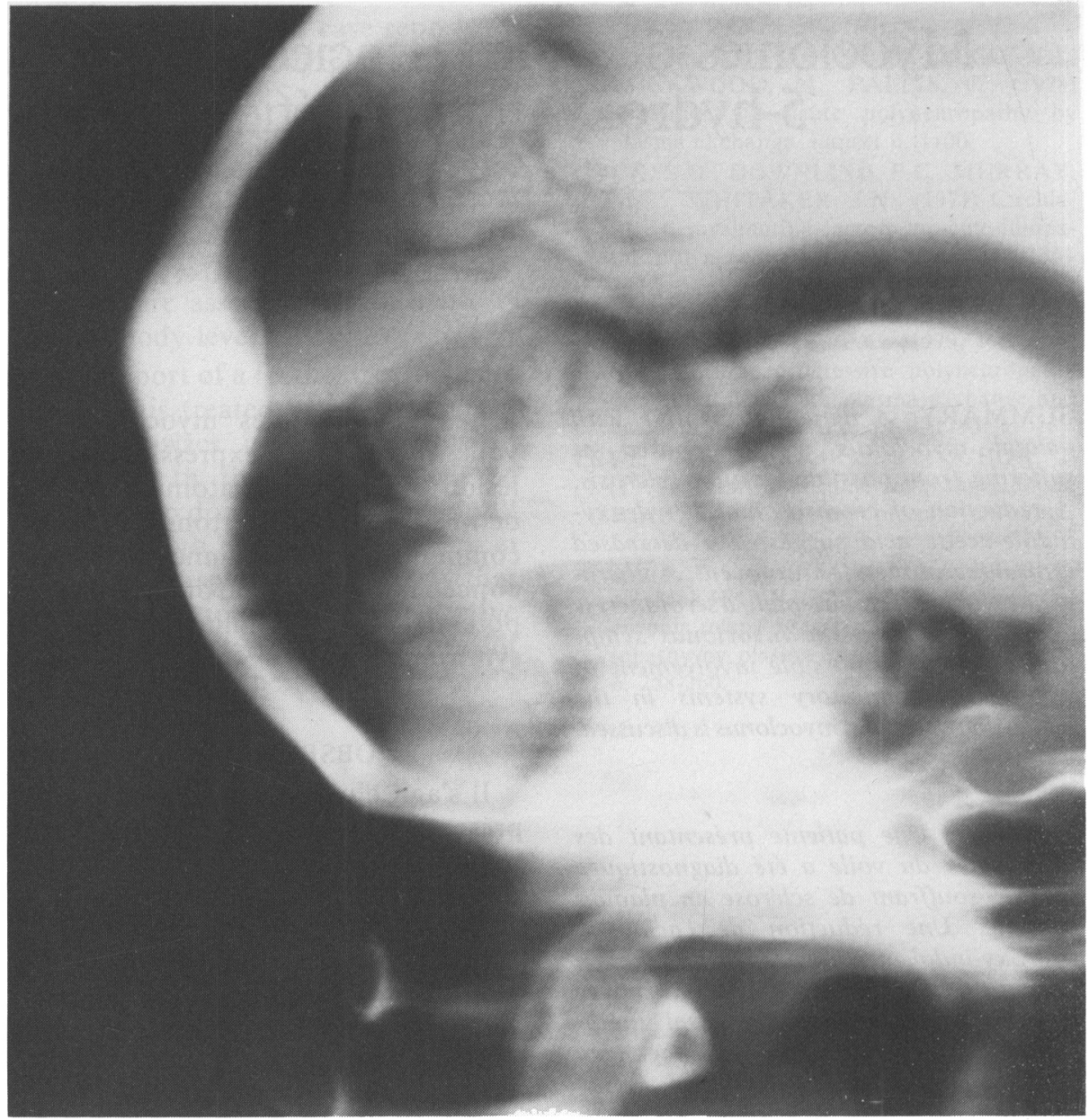

Figure 1 - Tomographie sagittale après injection d'oxygène par voie lombaire: atrophie midvermienne, sans anomalie du tronc cérébral.

TABLEAU 1

Niveaux de tryptophane et d'acide 5-Hydroxy-Indole-Acétique dans le LCR prélevé par encéphalographie gazeuse $(n g / m l \pm D S)$

\begin{tabular}{lccccc}
\hline & \multicolumn{2}{c}{ Niveau lombaire } & & \multicolumn{2}{c}{ Niveau cisternal } \\
\cline { 2 - 3 } \cline { 5 - 6 } \cline { 5 - 6 } & Tryptophane & 5HIAA & & Tryptophane & 5HIAA \\
Contrôles (19) & $379 \pm 113$ & $28.1 \pm 13.1$ & & $375 \pm 88$ & $33.3 \pm 9.5$ \\
Sclérose en plaques & 354 & 28.7 & & 426 & 48.7 \\
$\quad$ possible sans & 350 & 26.0 & & 355 & 29.6 \\
myoclonies du voile & 312 & 28.9 & & 227 & 24.9 \\
$\begin{array}{c}\text { Patiente avec } \\
\text { myoclonies du }\end{array}$ & 184 & 9.3 & & 219 & 29.9 \\
$\quad$ voile & & & & \\
\hline
\end{tabular}

anatomiques impliquées dans les myoclonies du voile, du noyau dentelé à l'olive inférieure contralatérale, à travers le pédoncule cérébelleux supérieur et le faisceau tegmentaire central contralatéral (Lapresle, 1979), traversent une région vulnérable aux plaques de démyélinisation (McAlpine et al., 1972). Il est de fait surprenant que les myoclonies du voile aient été rapportées 
si peu fréquemment dans la sclérose en plaques, et il est possible que des mécanismes effecteurs soient également atteints.

La patiente a subi une encéphalographie gazeuse pour éliminer une lésion expansive du tronc cérébral. Du LCR a été prélevé tel que décrit dans l'observation. Les niveaux de 5HIAA et de tryptophane étaient diminués au niveau lombaire, par rapport à des contrôles souffrant de divers troubles neurologiques non associés à des anomalies des amines cérébrales. Ces contrôles sont tous de sexe féminin et d'âge équivalent à la patiente, étant donné que nous avons déjà décrit une variation du niveau de SHIAA reliée au sexe et à l'âge (Young et al., 1980). Certains rapports ont déjà mis en évidence une diminution du 5HIAA lombaire chez des cas avancés de sclérose en plaques, mais des valeurs normales chez les patients récemment diagnostiqués (Claveria et al., 1974; Davidson et al., 1977; Soninen et al., 1973). Nous avons donc comparé le niveau obtenu chez cette patiente avec celui de trois femmes souffrant de sclérose en plaques possible, sans myoclonies du voile. Nous n'avons pu trouver une diminution de 5HIAA chez ces trois patientes. L'observation d'une réduction nette de 5HIAA associée aux myoclonies du voile a donc possiblement une signification particulière quant à la pathophysiologie de ces mouvements.

Chadwick et al (1977) ont trouvé des taux réduits de 5HIAA céphalorachidien chez certains patients souffrant de divers types de myoclonies. Le seul patient avec myoclonies du voile (patient 15) n'a pas eu de ponction lombaire. Il n'avait pas répondu à une administration aiguë de clonazépam, mais un traitement prolongé n'avait pas été entrepris. Le taux normal de 5HIAA dans l'échantillon cisternal de notre patiente suggère un turnover normal dans les régions contenant la masse des corps cellulaires sérotonergiques. Par contre, la réduction dans l'échantillon lombaire suggère une diminution de l'activité des projections bulbospinales sérotonergiques. La présence de lésions démyélinisantes spinales affectant ces projections a déjà été suggérée par Davidson et al
(1977) comme cause de la réduction de 5HIAA dans la sclérose en plaques avancée. Cependant, les axones de ces projections n'étant pas myélinisés (Dalhstrom et Fuxe, 1965), il est probable que des lésions sévères avec atteinte axonale, ou chroniques avec gliose marquée, soient nécessaires pour impliquer ces voies. Dans le cas qui nous occupe, il est possible que certaines projections sérotonergiques du raphé caudal à l'olive inférieure (Dalhstrom et Fuxe, 1965), soient impliquées dans les myoclonies du voile. Dans les types de myoclonies qu'ils ont étudiées, Chadwick et son groupe ont conclu en une réduction fonctionnelle de l'activité sérotonergique résultant en une libération de réflexes primitifs du tronc cérébral normalement inhibés. De la même façon, les myoclonies du voile, déjà comparées aux mouvements des branchies (Stern, 1949), pourraient être l'expression d'une telle désinhibition sérotonergique.

Une diminution du niveau de 5HIAA céphalo-rachidien peut également provenir d'une réduction du précurseur physiologique, le tryptophane. Bien que le niveau de tryptophane lombaire apparaît réduit chez notre patiente, il est improbable que ce facteur joue un rôle prédominant dans la réduction du tonus sérotonergique: la variabilité physiologique des niveaux céphalo-rachidiens de tryptophane est grande, et le taux de tryptophane au niveau cisternal est normal chez notre patiente. Enfin, la valeur de 5HIAA lombaire est réduite à $33 \%$ des valeurs contrôles par rapport à une réduction de $50 \%$ pour le tryptophane. Un rapport inverse aurait été nécessaire pour impliquer une diminution du précurseur affectant le niveau du transmetteur. Nous n'avons pas trouvé chez les patients souffrant de sclérose en plaques possible une diminution du tryptophane céphalo-rachidien, diminution que Monaco et al (1949) ont rapportée chez un groupe de patients souffrant de "sclérose en plaques définitive".

Magnussen et al (1977) ont décrit le cas d'un patient souffrant de myoclonies du voile, d'origine post-traumatique, amélioré par la combinaison de 5-hydroxytryptophane et carbidopa.
A la suite de cette observation, et considérant le niveau diminué de 5HIAA céphalo-rachidien chez notre patiente, nous avons choisi de la traiter avec le clonazépam, qui semble avoir un mode d'action sérotonergique (Chadwick et al., 1977; Chung Hwang et Vanwoert, 1979), bien qu'une facilitation de la transmission glycinergique (Young et al., 1974) ait déjà été suggérée. Cette médication a été très bien tolérée par la patiente, et la réponse clinique très rapide, avec amélioration symptomatique. Les myoclonies ayant été présentes depuis 5 ans sans fluctuations notables, nous considérons que l'amélioration observée est véritablement un effet médicamenteux et non une simple variation biologique d'un symptôme neurologique.

\section{CONCLUSION}

Les myoclonies du voile chez notre patiente semblent associées à une réduction du turnover sérotonergique central, tel que reflété par le taux de 5HIAA céphalo-rachidien. Etant donné l'amélioration clinique consécutive au traitement par le clonazépam, nous pouvons confirmer les conclusions du Chadwick et al (1977) sur la valeur du dosage de ce métabolite chez les patients souffrant de myoclonies: une réduction du 5HIAA prédit habituellement une bonne réponse clinique aux agonistes sérotonergiques.

\section{BIBLIOGRAPHIE}

ANDERSON, G.M. et PURDY, W.C. (1979) A liquid chromatographic-fluorometric system for the analysis of indoles in physiological samples. Anal. Chem., 51, 283286.

CHADWICK, D., HALLETT, M., HARRIS, R., JENNER, P., REYNOLDS, E.H. et MARSDEN, C.D. (1977). Clinical, biochemical, and physiological features distinguishing myoclonus responsive to 5 hydroxytryptophan, tryptophan with a monoamine oxydase inhibitor, and clonazepam. Brain, 100, 455-487.

CHUNG HWANG, E. et VANWOERT, M.H (1979). Antimyoclonic action of clonazepam the role of serotonin. Eur. Pharmacol., 60 31-40.

CLAVERIA, L.E., CURZON, G., HARRISON, M.J.G. et KANTAMANONI, B.D. (1974). Amine metabolites in the cerebrospinal fluid of patients with disseminated sclerosis. J. Neurol., 37, 715-718.

DALHSTROM, A. et FUXE, K. (1965). Evidence for the existence of monoamine 
neurons in the central nervous system. Acta Physiol. Scand., 65. supplément 247.

DAVIDSON, D., PULLAR, I.A., MAWDSLEY, C., KINLOCH, N. et YATES, C.M. (1977). Monoamine metabolites in cerebrospinal fluid in multiple sclerosis. J. Neurol. Neurosurg. Psychiatry, 40, 741-745.

LAPRESLE, J. (1979). Rhythmic palatal myoclonus and the dentalo-olivary pathway. J. Neurol., 220, 223-230.

MAGNUSSEN, I., DUPONT. E., PRANGUEHANSEN, A. et De FINE OLIVARIUS, B. (1977). Palatal myoclonus treated with 5-hydroxytryptophan and a decarboxylaseinhibitor. Acta Neurol. Scand., 55, 251-253.

MCALPINE, D.J., LUMSDEN, C.E. et
ACHESON, E.D. (1972). Multiple sclerosis: a reappraisal. Livingston. Edinburgh \& London.

MONACO, F., FUMERO, S., MONDINO, A. et MUTANI. R. (1949). Plasma and cerebrospinal fluid tryptophan in multiple sclerosis and degenerative diseases. J. Neurol. Neurosurg. Psychiatry, 42, 640-641.

RONDOT, P. et BEN HAMIDA, M. (1968). Myoclonies du voile et myoclonies squelettiques, Etude clinique et anatomique. Rev. Neurol., 119, 59-83.

SONNINEN, V., RIEKKINEN, P. et RINNE, H.K. (1973). Acid monoamine metabolites in cerebrospinal fluid in multiple sclerosis. Neurology, 23, 760-763.
STERN, M.M. (1949), Rhythmic palatopharyngeal myoclonus. Review, case report and significance. J. Nerv. Ment. Dis., 109, 48-53.

YOUNG, A.B., ZUKIN, S.R. et SNYDER, S.H. (1974). Interaction of benzodiazepines with central nervous glycine receptors: possible mechanism of actions. Proc. Natl. Acad. Sci., 71, 2246-2250.

YOUNG, S.N., GAUTHIER,S., ANDERSON, G.M. et PURDY, W.C. (1980). Tryptophan, 5-hydroxyindole-acetic acid and indoleacetic acid in human cerebrospinal fluid; interrelationships and the influence of age, sex. epilepsy and anticonvulsant drugs. J, Neurol. Neurosurg. Psychiatry, 43, 438-445. 